\title{
PERTURBATIONS D'EXEMPLES DE LATTĖS ET DIMENSION DE HAUSDORFF DU LIEU DE BIFURCATION
}

\author{
FRANÇOIS BERTELOOT AND FABRIZIO BIANCHI
}

\begin{abstract}
We give an estimate for the Hausdorff dimension of the bifurcation locus of a family of endomorphisms of $\mathbb{P}^{k}(\mathbb{C})$. This dimension is maximal near isolated Lattès examples.
\end{abstract}

\section{INTRODUCTION}

Un exemple de Lattès de degré algébrique $d$ est un endomorphisme holomorphe $f$ de $\mathbb{P}^{k}$ faisant commuter un diagramme

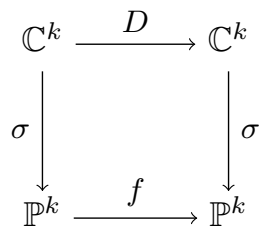

où $D$ est une application affine de partie linéaire $\sqrt{d} U$ ( $U$ unitaire) et $\sigma$ est un revêtement ramifié sur les fibres duquel un groupe cristallographique complexe agit transitivement. Ces exemples ont étés découverts en 1918 par $\mathrm{S}$. Lattès pour la dimension $k=1$ [Lat] mais ils existent en toute dimension et tout degré. L'article de J. Milnor [Mil] décrit ces objets d'un point de vue contemporain. La caractérisation suivante, due à A. Zdunik $[\mathrm{Zdu}$ en dimension $k=1$ et F. Berteloot, C. Dupont et J.J. Loeb $[\mathrm{BL}, \overline{\mathrm{BeDu}}, \mathrm{Dup}$ en toute dimension, permet d'amorcer l'étude des bifurcations engendrées par l'extrème rigidité de ces endomorphismes.

Théorème 1.1. Soit $f$ un endomorphisme holomorphe de degré algébrique $d$ sur $\mathbb{P}^{k}$ et $\mu_{f}$ sa mesure d'entropie maximale. Les assertions suivantes sont équivalentes :

1) $\mu_{f}$ est absolument continue par rapport à la mesure de Lebesgue,

2) les exposants de Lyapounov de $\left(f, \mu_{f}\right)$ sont minimaux égaux à $\ln \sqrt{d}$,

3) $f$ est un exemple de Lattès.

Une famille holomorphe d'endomorphismes de $\mathbb{P}^{k}$, paramétrée par une variété complexe $M$, est une application holomorphe $F: M \times \mathbb{P}^{k} \rightarrow M \times \mathbb{P}^{k}$ de la forme $\left(\lambda, f_{\lambda}(z)\right)$ et telle que le degré algébrique des endomorphismes $f_{\lambda}$ soit égal à $d$ pour tout $\lambda \in M$. En dimension $k=1$, la nature des bifurcations au sein de telles familles est bien comprise depuis les travaux fondateurs de R. Mañé, P. Sad et D. Sullivan [MSS] et, indépendemment, M. Lyubich [Ly1],[Ly2]. De plus, comme l'a montré L. DeMarco deM, le lieu de bifurcation $\operatorname{Bif}(F)$ coïncide avec le support du $(1,1)$-courant positif fermé $d d^{c} L(\lambda)$ où $L(\lambda)$ désigne l'exposant de Lyapounov de $f_{\lambda}$ par rapport à sa mesure d'entropie maximale. Dans un travail en collaboration avec C. Dupont, les auteurs ont

This research was partially supported by the ANR project LAMBDA, ANR-13-BS01-0002. 
étendu cette théorie en dimension arbitraire. Dans ce contexte la fonction $L(\lambda)$ désigne la somme des exposants de Lyapounov de $f_{\lambda}$ par rapport à sa mesure d'entropie maximale $\mu_{\lambda}$, l'ensemble de Julia $J(\lambda)$ de $f_{\lambda}$ est par définition le support de $\mu_{\lambda}$ et un $J$-cycle est un cycle contenu dans $J(\lambda)$. Le principal résultat est le suivant, nous renvoyons à [BBD] pour un énoncé plus complet.

Théorème 1.2. Soit $F: M \times \mathbb{P}^{k} \rightarrow M \times \mathbb{P}^{k}$ une famille holomorphe d'endomorphismes où $M$ est un ouvert simplement connexe de l'espace des endomorphismes de $\mathbb{P}^{k}$ de degré $d \geq 2$. Alors les assertions suivantes sont équivalentes :

1) les $J$-cycles répulsifs de $f_{\lambda}$ bougent holomorphiquement sur $M$,

2) la fonction $L$ est pluriharmonique sur $M$,

3) $J(\lambda)$ bouge holomorphiquement sur $M$.

On définit alors le lieu de bifurcation $\operatorname{Bif}(F)$ d'une telle famille comme le support du $(1,1)$ courant positif fermé $d d^{c} L(\lambda)$. Il est remarquable qu'en dimension $k \geq 2$ (et contrairement au cas de la dimension $k=1$ ) le lieu de bifurcation puisse être d'intérieur non vide. Ce phénomène a été récemment mis en évidence par le deuxième auteur et J. Taflin [BiTa et R. Dujardin [Duj].

Lorsque $f_{\lambda_{0}}$ est un exemple de Lattès isolé on voit, grâce à la caractérisation par la minimalité de la somme des exposants de Lyapounov, que $\lambda_{0}$ est un paramètre de bifurcation. Nous pensons que ces endomorphismes sont en fait le foyer de bifurcations maximales qu'il conviendrait d'étudier.

Illustrons ceci en rappelant ce qui est connu en dimension $k=1$. Comme l'ont remarqué G. Bassanelli et F. Berteloot, l'argument de minimalité de l'exposant montre aussi que $\lambda_{0}$ est dans le support des puissances extérieures de $d d^{c} L$ ce qui permet de préciser le nombre de cycles répulsifs bifurquants simultanément. Par exemple, dans l'espace des fractions rationnelles de degré $d$, on peut montrer qu'un exemple de Lattès non flexible est à la fois accumulé par des fractions rationnelles hyperboliques et par des fractions rationnelles possédant $2 d-2$ cycles neutres distincts ([BB], voir aussi [Ber] subsection 6.2.3). Ceci a été étendu aux exemples de Lattès flexibles par X. Buff et T. Gauthier BuGa. Un résultat important de M. Shishikura stipule qu'une fraction de degré $d$ possède au plus $2 d-2$ cycles non répulsifs et que cette borne est réalisée [Sh1]. Les bifurcations de ce type sont donc, en un certain sens, maximales. Soulignons que T. Gauthier [Gau a montré que la dimension de Hausdorff du lieu des bifurcations maximales est elle-même maximale.

Nous initions dans cet article l'étude des bifurcations engendrées par un exemple de Lattès en dimension quelconque. Nous montrons que le lieu de ces bifurcations est de dimension de Hausdorff maximale dans "toutes les directions". Notre principal résultat est le suivant.

Théorème 1.3. Soit $F: D \times \mathbb{P}^{k} \rightarrow D \times \mathbb{P}^{k}$ une famille holomorphe d'endomorphismes de degré $d \geq 2$ paramétrée par le disque unité $D$ de $\mathbb{C}$. On suppose que $f_{0}$ est un exemple de Lattès et que 0 est accumulé par des paramètres $\lambda \in D$ tel que $f_{\lambda}$ n'est pas un exemple de Lattès. Alors $\operatorname{dim}_{H}(\operatorname{Bif}(F))=2$.

Terminons cette introduction en précisant quelques notations.

Notations 1.4. $D$ est le disque unité de $\mathbb{C}$ et $D_{r}$ désigne le disque $r D$.

Pour tout sous-ensemble $E$ d'un produit $D \times B$ et tout $\lambda \in D$, on note $(E)_{\lambda}$ la tranche $E \cap(\{\lambda\} \times B)$. On note $\pi_{D}$ la projection canonique sur $D$.

On note $\Gamma_{\gamma}:=\{(\lambda, \gamma(\lambda)): \lambda \in D\}$ le graphe d'une application $\gamma: D \rightarrow B$.

$\mathcal{O}(D, B)$ est l'espace des applications holomorphes de $D$ dans $B$.

$\mathcal{H}_{d}\left(\mathbb{P}^{k}\right)$ est l'espace des endomorphismes holomorphes de degré algébrique d sur $\mathbb{P}^{k}$. 
PERTURBATIONS D'EXEMPLES DE LATTÈS ET DIMENSION DE HAUSDORFF DU LIEU DE BIFURCATION3

\section{LAMINATIONS ENGENDRÉES PAR DES CONTRACTIONS}

Dans toute cette section, $B$ désignera une boule de $\mathbb{C}^{k}$ pour la norme hermitienne standard. Rappelons que le disque unité de $\mathbb{C}$ est noté $D$. Nous allons construire une lamination dans $D \times B$ par une famille $\mathcal{G}$ de graphes holomorphes au-dessus de $D$ dont les tranches sont des ensembles de Cantor. La dimension de Hausdorff des tranches sera minorée en utilisant les travaux de Pesin et Weiss [PW] sur les constructions géométriques du type Moran (voir aussi [Pes, Chapter 5]) .

L'ensemble $\mathcal{G}$ est obtenu par un procédé usuel à partir d'une collection $G_{1}, \cdots, G_{m}$ de contractions holomorphes de la forme $G_{j}(\lambda, z)=\left(\lambda, G_{j, \lambda}(z)\right)$, définies sur un voisinage $\widetilde{D} \times \widetilde{B}$ de $\bar{D} \times \bar{B}$ et vérifiant les propriétés suivantes pour des constantes $0<a \leq A$

1) $G_{j}(\bar{D} \times \bar{B}) \subset \bar{D} \times B, \forall j \in\{1, \cdots, m\}$

2) $\operatorname{dist}\left(G_{j}(\bar{D} \times \bar{B}), G_{k}(\bar{D} \times \bar{B})\right)>0, \forall j \neq k \in\{1, \cdots, m\}$

3) $e^{-A}\left\|z-z^{\prime}\right\| \leq\left\|G_{j}(\lambda, z)-G_{j}\left(\lambda, z^{\prime}\right)\right\| \leq e^{-a}\left\|z-z^{\prime}\right\|, \forall \lambda \in \bar{D}, \forall z, z^{\prime} \in \bar{B}, \forall j \in\{1, \cdots, m\}$.

Pour tout $\omega:=\left(\omega_{k}\right)_{k \geq 0} \in\{1, \ldots, m\}^{\mathbb{N}}=: \Sigma_{m}^{+}$, on pose $G_{\omega_{0} \cdots \omega_{p}}:=G_{\omega_{0}} \circ \cdots \circ G_{\omega_{p}}$ puis

$$
\begin{aligned}
T_{\omega_{0} \cdots \omega_{p}} & :=G_{\omega_{0} \cdots \omega_{p}}(\bar{D} \times \bar{B}) \\
\Gamma_{\omega} & :=\cap_{p \geq 0} T_{\omega_{0} \cdots \omega_{p}} .
\end{aligned}
$$

On observera que les applications $G_{\omega_{0} \cdots \omega_{p}}$ sont de la forme $(\lambda, z) \mapsto\left(\lambda, G_{\omega_{0} \cdots \omega_{p}, \lambda}(z)\right)$ et que

$$
\left\|G_{\omega_{0} \cdots \omega_{p}}(\lambda, z)-G_{\omega_{0} \cdots \omega_{p}}\left(\lambda, z^{\prime}\right)\right\| \leq e^{-(p+1) a}\left\|z-z^{\prime}\right\|, \forall \lambda \in \bar{D}, \forall z, z^{\prime} \in \bar{B}
$$

En particulier, $\left(T_{\omega_{0} \cdots \omega_{p}}\right)_{\lambda}$ est une suite décroissante de compacts dont le diamètre tend vers 0 lorsque $p$ tend vers $+\infty$ et il existe donc un unique point $\omega(\lambda) \in B$ tel que

$$
\left(\cap_{p \geq 0} T_{\omega_{0} \cdots \omega_{p}}\right)_{\lambda}=\cap_{p \geq 0}\left(T_{\omega_{0} \cdots \omega_{p}}\right)_{\lambda}=\{(\lambda, \omega(\lambda))\} .
$$

Autrement dit, $\Gamma_{\omega}$ est le graphe d'une application définie sur $\bar{D}$ et à valeurs dans $B$ que l'on note aussi $\omega$. On notera $\mathcal{G}$ la réunion des graphes ainsi obtenus

$$
\mathcal{G}:=\bigcup_{\omega \in \Sigma_{m}^{+}} \Gamma_{\omega}
$$

Proposition 2.1. L'ensemble $\mathcal{G}$ est constitué de graphes deux à deux disjoints, continus sur $\bar{D}$ et holomorphes sur D. Pour tout $\lambda_{0} \in D$, la dimension de Hausdorff de $(\mathcal{G})_{\lambda_{0}}$ est minorée par $\frac{\ln m}{A}$ et l'application $H_{\lambda_{0}}: \mathcal{G} \rightarrow(\mathcal{G})_{\lambda_{0}}$ définie par $H_{\lambda_{0}}(\lambda, \omega(\lambda))=\left(\lambda_{0}, \omega\left(\lambda_{0}\right)\right)$ est $\frac{a}{A}$-Hölder sur $\mathcal{G} \cap\left(D_{r} \times B\right)$ pour tout $0<r<1$.

DÉmonstration: Fixons $z_{0} \in B$. L'inégalité (1) appliquée à $z^{\prime}=G_{\omega_{p+1} \cdots \omega_{p+q}}\left(\lambda, z_{0}\right)$ et $z=z_{0}$ donne

$$
\left\|G_{\omega_{0} \cdots \omega_{p}}\left(\lambda, z_{0}\right)-G_{\omega_{0} \cdots \omega_{p+q}}\left(\lambda, z_{0}\right)\right\| \leq e^{-(p+1) a} \operatorname{diam}(B), \forall \lambda \in \bar{D} .
$$

Les propriétés de régularité de $\omega$ résultent alors de la convergence uniforme sur $\bar{D}$ de $G_{\omega_{0} \cdots \omega_{p}}\left(\lambda, z_{0}\right)$ $\operatorname{vers}(\lambda, \omega(\lambda))$.

Nous allons maintenant montrer que pour tout $0<r<1$ il existe une constante $C_{r}>0$ telle que si $\left(\omega_{0}, \cdots, \omega_{p}\right) \neq\left(\omega_{0}^{\prime}, \cdots, \omega_{p}^{\prime}\right)$ alors

$$
\left\|G_{\omega_{0} \cdots \omega_{p}}(\lambda, z)-G_{\omega_{0}^{\prime} \cdots \omega_{p}^{\prime}}\left(\lambda^{\prime}, z^{\prime}\right)\right\| \geq C_{r} e^{-p A}, \forall(\lambda, z),\left(\lambda^{\prime}, z^{\prime}\right) \in D_{r} \times B .
$$


Puisque $G_{\omega_{0} \cdots \omega_{p}}(\bar{D} \times \bar{B}) \subset \bar{D} \times B$, on déduit des inégalités de Cauchy qu'il existe une constante $K_{r} \geq 1$ indépendante de $\left(\omega_{0}, \cdots, \omega_{p}\right)$ telle que

$$
\left\|G_{\omega_{0} \cdots \omega_{p}}(\lambda, z)-G_{\omega_{0} \cdots \omega_{p}}\left(\lambda^{\prime}, z\right)\right\| \leq K_{r}\left|\lambda-\lambda^{\prime}\right|, \forall(\lambda, z),\left(\lambda^{\prime}, z\right) \in D_{r} \times \bar{B} .
$$

Soit $0<d:=\min _{1 \leq j \neq k \leq m} \operatorname{dist}\left(G_{j}(\bar{D} \times \bar{B}), G_{k}(\bar{D} \times \bar{B})\right)$. Soit $(\lambda, z),\left(\lambda, z^{\prime}\right) \in D_{r} \times \bar{B}$, comme $\left\|G_{\omega_{j}}(\lambda, z)-G_{\omega_{j}^{\prime}}\left(\lambda, z^{\prime}\right)\right\| \geq e^{-A}\left\|z-z^{\prime}\right\|$ si $\omega_{j}=\omega_{j}^{\prime}$ et $\left\|G_{\omega_{j}}(\lambda, z)-G_{\omega_{j}^{\prime}}\left(\lambda, z^{\prime}\right)\right\| \geq d$ sinon, on voit que

$$
\left\|G_{\omega_{0} \cdots \omega_{p}}(\lambda, z)-G_{\omega_{0}^{\prime} \cdots \omega_{p}^{\prime}}\left(\lambda, z^{\prime}\right)\right\| \geq e^{-p A} d, \quad \forall(\lambda, z),\left(\lambda, z^{\prime}\right) \in D_{r} \times B .
$$

Supposons que $\left|\lambda-\lambda^{\prime}\right| \leq \frac{d}{2 K_{r}} e^{-p A}$, on déduit alors de (4) et (5) que la minoration $\| G_{\omega_{0} \cdots \omega_{p}}(\lambda, z)-$ $G_{\omega_{0}^{\prime} \cdots \omega_{p}^{\prime}}\left(\lambda^{\prime}, z^{\prime}\right) \| \geq e^{-p A} d-K_{r}\left|\lambda-\lambda^{\prime}\right| \geq \frac{d}{2} e^{-p A} \geq \frac{d}{2 K_{r}} e^{-p A}$ a lieu pour tout $z, z^{\prime} \in B$. Comme $\left\|G_{\omega_{0} \cdots \omega_{p}}(\lambda, z)-G_{\omega_{0}^{\prime} \cdots \omega_{p}^{\prime}}\left(\lambda^{\prime}, z^{\prime}\right)\right\| \geq\left|\lambda-\lambda^{\prime}\right|$, la même minoration reste vraie lorsque $\left|\lambda-\lambda^{\prime}\right| \geq$ $\frac{d}{2 K_{r}} e^{-p A}$. Ceci justifie la minoration (3) avec $C_{r}:=\frac{d}{2 K_{r}}$.

Nous pouvons maintenant terminer la preuve de la proposition. Soit $\lambda_{0} \in D$, l'inégalité (3) montre en particulier qu'il existe une constante $C>0$ que

$$
\operatorname{dist}\left(\left(T_{\omega_{0} \cdots \omega_{p}}\right)_{\lambda_{0}},\left(T_{\omega_{0}^{\prime} \cdots \omega_{p}^{\prime}}\right)_{\lambda_{0}}\right) \geq C e^{-p A} \text { si }\left(\omega_{0}, \cdots, \omega_{p}\right) \neq\left(\omega_{0}^{\prime}, \cdots, \omega_{p}^{\prime}\right) .
$$

Un théorème dû à Pesin et Weiss (voir [PW], Proposition 5) stipule que dans ces conditions $\operatorname{dim}_{H}\left((\mathcal{G})_{\lambda_{0}}\right) \geq \frac{\ln m}{A}$.

Il nous reste à étudier la régularité de l'application $H_{\lambda_{0}}$. Soient $\omega \neq \omega^{\prime} \in \Sigma_{m}^{+}$et $p:=$ $\min \left\{j: \omega_{j} \neq \omega_{j}^{\prime}\right\}$. Fixons $z_{0} \in B$ et, pour $q>p$, posons $(\lambda, z):=G_{\omega_{p+1} \cdots \omega_{q}}\left(\lambda, z_{0}\right),\left(\lambda^{\prime}, z^{\prime}\right):=$ $G_{\omega_{p+1}^{\prime} \cdots \omega_{q}^{\prime}}\left(\lambda^{\prime}, z_{0}\right)$. D'après (3) il vient $\left\|G_{\omega_{0} \cdots \omega_{q}}\left(\lambda, z_{0}\right)-G_{\omega_{0}^{\prime} \cdots \omega_{q}^{\prime}}\left(\lambda^{\prime}, z_{0}\right)\right\|=\left\|G_{\omega_{0} \cdots \omega_{p}}(\lambda, z)-G_{\omega_{0}^{\prime} \cdots \omega_{p}^{\prime}}\left(\lambda^{\prime}, z^{\prime}\right)\right\| \geq$ $C_{r} e^{-p A}$ pour tout $\lambda, \lambda^{\prime} \in D_{r}$ d'où, en faisant tendre $q$ vers $+\infty$,

$$
\left\|(\lambda, \omega(\lambda))-\left(\lambda^{\prime}, \omega^{\prime}\left(\lambda^{\prime}\right)\right)\right\| \geq C_{r} e^{-p A}, \quad \forall \lambda, \lambda^{\prime} \in D_{r} .
$$

Par ailleurs, puisque $\left(\lambda_{0}, \omega\left(\lambda_{0}\right)\right)$ et $\left(\lambda_{0}, \omega^{\prime}\left(\lambda_{0}\right)\right)$ sont tous deux dans $G_{\omega_{0} \cdots \omega_{p-1}}(D \times B)$, on a

$$
\left\|\omega\left(\lambda_{0}\right)-\omega^{\prime}\left(\lambda_{0}\right)\right\| \leq e^{-p a}(\operatorname{diam} B) .
$$

On tire de (6) et (7) que $\left\|\omega\left(\lambda_{0}\right)-\omega^{\prime}\left(\lambda_{0}\right)\right\| \leq C_{r}^{-\frac{a}{A}}(\operatorname{diam} B)\left\|(\lambda, \omega(\lambda))-\left(\lambda^{\prime}, \omega^{\prime}\left(\lambda^{\prime}\right)\right)\right\|^{\frac{a}{A}}$ pour $\lambda, \lambda^{\prime} \in D_{r}$ ce qui signifie que l'application $H_{\lambda_{0}}$ est $\frac{a}{A}$-Hölder sur $\mathcal{G} \cap\left(D_{r} \times B\right)$.

Supposons maintenant que $D \times B$ contient une hypersurface irreductible $Z$ qui n'est pas réduite à une fibre de $\pi_{D}$ et pour laquelle $\pi_{D}(Z) \subset D_{r_{0}}$ où $0<r_{0}<1$. Nous allons minorer $\operatorname{dim}_{H} \pi_{D}(\mathcal{G} \cap Z)$. Commençons par observer que tous les graphes de $\mathcal{G}$ intersectent $Z$.

Lemme 2.2. L'intersection $\Gamma_{\gamma} \cap Z$ est non vide pour tout $\gamma \in \mathcal{O}(D, B)$. En particulier, $\Gamma_{\omega} \cap Z$ est non vide et discret pour tout $\omega \in \Sigma_{m}^{+}$.

DÉmonstration: L'espace $\mathcal{O}(D, B)$ est convexe, on le munit de la topologie de la convergence uniforme locale. Pour tout $\gamma \in \mathcal{O}(D, B)$, le sous-ensemble analytique $\pi_{D}\left(\Gamma_{\gamma} \cap Z\right)$ de $D$ est de dimension nulle car il est relativement compact dans $D$. Ainsi $\Gamma_{\gamma} \cap Z$ est discret (ou vide). L'ensemble $\left\{\gamma \in \mathcal{O}(D, B): \Gamma_{\gamma} \cap Z \neq \emptyset\right\}$ est clairement fermé dans $\mathcal{O}(D, B)$, le lemme d'Hurwitz montre qu'il est ouvert et la conclusion s'ensuit. 
Proposition 2.3. Pour toute hypersurface irreductible $Z \subset D \times B$, non réduite à une fibre de $\pi_{D}$ et telle que $\pi_{D}(Z) \Subset D$ on a l'estimation suivante : $\operatorname{dim}_{H} \pi_{D}(\mathcal{G} \cap Z) \geq \frac{a}{A}\left(\frac{\ln m}{A}\right)-(2 k-2)$.

DÉmonstration: On suppose que $\frac{\ln m}{A}>\frac{A}{a}(2 k-2)$ car sinon il n'y a rien à démontrer. Voyons d'abord comment se ramener au cas où $Z$ est lisse et transverse aux fibres de $\pi_{D}$. Rappelons que $Z$ est un sous-ensemble analytique de dimension complexe $k$ dans $D \times B$. Soit $S$ l'ensemble constitué des points singuliers de $Z$ ainsi que de ses points réguliers $p$ pour lesquels l'espace tangent $T_{p}(Z)$ est confondu avec la fibre $\pi_{D}^{-1}\left(\pi_{D}(p)\right)$. Nous allons montrer qu'il existe $\tilde{\omega} \in \Sigma_{m}^{+}$tel que $\Gamma_{\tilde{\omega}} \cap Z$ n'est pas contenu dans $S$.

Comme $S$ est un sous-ensemble analytique strict de $Z$ (par hypothèse $Z$ n'est pas une fibre de $\pi_{D}$ ) on a $\operatorname{dim}_{H}(S) \leq 2 k-2$. Supposons que $\Gamma_{\omega} \cap Z \subset S$ pour tout $\omega \in \Sigma_{m}^{+}$et fixons $\lambda_{0} \in D$. Comme $\Gamma_{\omega} \cap Z \neq \emptyset$ on a $H_{\lambda_{0}}\left(\Gamma_{\omega}\right)=H_{\lambda_{0}}\left(\Gamma_{\omega} \cap Z\right)$ pour tout $\omega \in \Sigma_{m}^{+}$et donc $(\mathcal{G})_{\lambda_{0}}=H_{\lambda_{0}}(\mathcal{G})=\cup_{\omega \in \Sigma_{m}^{+}} H_{\lambda_{0}}\left(\Gamma_{\omega}\right) \subset H_{\lambda_{0}}(S)$. D'après la Proposition 2.1 cela entraîne que $\frac{\ln m}{A} \leq \operatorname{dim}_{H}\left((\mathcal{G})_{\lambda_{0}}\right) \leq \frac{A}{a} \operatorname{dim}_{H}(S) \leq \frac{A}{a}(2 k-2)$ ce qui est exclu. Il existe donc $\tilde{\omega} \in \Sigma_{m}^{+}$et $\lambda_{1} \in D_{r_{0}}$ tels que $\left(\lambda_{1}, \tilde{\omega}\left(\lambda_{1}\right)\right) \in Z \backslash S$. Etant donné un voisinage $V$ de $\left(\lambda_{1}, \tilde{\omega}\left(\lambda_{1}\right)\right)$ dans $Z \backslash S$ on voit, grâce au lemme d'Hurwitz, que si $p$ est assez grand alors $\Gamma_{\omega} \cap V \neq \emptyset$ pour tout $\omega \in \Sigma_{m}^{+}$ vérifiant $\omega_{j}=\tilde{\omega}_{j}$ pour $j \leq p$.

Nous obtiendrons l'estimation annoncée en remplaçant $\mathcal{G}$ par $\widetilde{\mathcal{G}}_{p}:=\cup_{\omega \in \widetilde{C}_{p}} \Gamma_{\omega}$ où $\widetilde{C}_{p}:=\{\omega \in$ $\Sigma_{m}^{+}: \omega_{j}=\tilde{\omega}_{j}$ si $\left.j \leq p\right\}$. Observons que $\left(\widetilde{\mathcal{G}}_{p}\right)_{\lambda}=G_{\tilde{\omega}_{0} \ldots \tilde{\omega}_{p}}\left((\mathcal{G})_{\lambda}\right)$ pour tout $\lambda \in D$. Comme $G_{\tilde{\omega}_{0} \cdots \tilde{\omega}_{p}}$ est bi-lipschitzienne sur $\left\{\lambda_{0}\right\} \times B$, il résulte de la Proposition 2.1 que $\operatorname{dim}_{H}\left(\left(\widetilde{\mathcal{G}}_{p}\right)_{\lambda_{0}}\right) \geq \frac{\ln m}{A}$ puis, comme $\left(\widetilde{\mathcal{G}}_{p}\right)_{\lambda_{0}}=H_{\lambda_{0}}\left(\widetilde{\mathcal{G}}_{p}\right) \subset H_{\lambda_{0}}\left(\widetilde{\mathcal{G}}_{p} \cap Z\right)$, que $\frac{\ln m}{A} \leq \frac{A}{a} \operatorname{dim}_{H}\left(\widetilde{\mathcal{G}}_{p} \cap Z\right)$. Posons $E_{p}:=\widetilde{\mathcal{G}}_{p} \cap Z$; il nous reste pour conclure à justifier la majoration $\operatorname{dim}_{H}\left(E_{p}\right) \leq \operatorname{dim}_{H}\left(\pi_{D}(\mathcal{G} \cap Z)\right)+(2 k-2)$.

On peut supposer que $V=\{(l(z), z): z \in U\}$ où $U$ est un voisinage assez petit de $\tilde{\omega}\left(\lambda_{1}\right)$ et $l \in \mathcal{O}(U, \mathbb{C})$ vérifie $\frac{\partial l}{\partial z_{1}} \neq 0$ sur $U$. Alors, quitte à diminuer $U$, l'application $\psi(\lambda, z):=$ $\left(\lambda, l(z), z_{2}, \cdots, z_{k}\right)$ induit un biholomorphisme sur un voisinage $\Omega_{\lambda_{1}} \times U$ de $\left(\lambda_{1}, \tilde{\omega}\left(\lambda_{1}\right)\right)$ et, puisque $E_{p} \Subset \Omega_{\lambda_{1}} \times U$ pour $p$ est assez grand, il vient $\operatorname{dim}_{H}\left(E_{p}\right)=\operatorname{dim}_{H}\left(\psi\left(E_{p}\right)\right)$. On termine en remarquant que $\psi\left(E_{p}\right) \subset\left\{(\lambda, \lambda): \lambda \in \pi_{D}\left(E_{p}\right)\right\} \times \mathbb{C}^{k-1}$ et $\operatorname{dim}_{H}\left(\left\{(\lambda, \lambda): \lambda \in \pi_{D}\left(E_{p}\right)\right\} \times \mathbb{C}^{k-1}\right) \leq$ $\operatorname{dim}_{H}\left(\left\{(\lambda, \lambda): \lambda \in \pi_{D}\left(E_{p}\right)\right\}\right)+(2 k-2) \leq \operatorname{dim}_{H}\left(\pi_{D}(\mathcal{G} \cap Z)\right)+(2 k-2)$.

\section{Contractions issues D'une Perturbation dans $\mathcal{H}_{d}\left(\mathbb{P}^{k}\right)$}

Considérons $f \in \mathcal{H}_{d}\left(\mathbb{P}^{k}\right)$ dont les exposants de Lyapounov $\chi_{1} \leq \cdots \leq \chi_{k}$ relatifs à sa mesure d'entropie maximale $\mu_{f}$ ne satisfont aucune relation de résonnance (i.e. $\alpha_{1} \chi_{1}+\cdots+\alpha_{k} \chi_{k} \neq \chi_{j}$ pour tout $1 \leq j \leq k$ et tout $\alpha \in \mathbb{N}^{k}$ tel que $\alpha_{1}+\cdots+\alpha_{k} \geq 2$ ). Notre objectif est d'associer une lamination du type de celles étudiées à la section précédente à toute famille holomorphe $F: D \times \mathbb{P}^{k} \rightarrow D \times \mathbb{P}^{k}$ telle que $F(0, \cdot)=f$. Nous construirons pour cela des branches inverses itérées de $f$ dont les distorsions sont contrôlées puis les prolongerons en des branches inverses de $F$. Ceci repose essentiellement sur l'application à $f$ d'une méthode de linéarisation le long des orbites établie dans $[\mathrm{BDM}]$ et dont nous allons commencer par rappeler le principe.

Soit $\mathrm{O}:=\left\{\hat{x}:=\left(x_{n}\right)_{n \in \mathbb{Z}}: f\left(x_{n}\right)=x_{n+1}\right\}$ l'espace des orbites. On note $\pi$ la projection $\hat{x} \mapsto x_{0}$ puis $\hat{f}$ le décalage à gauche $(\pi \circ \hat{f}=f \circ \pi)$ et $\tau$ son inverse. Pour tout $E \subset \mathbb{P}^{k}$ on posera $\widehat{E}:=\pi^{-1}(E)$. Il existe une unique mesure de probabilité $\nu$ sur $\mathrm{O}$ telle que $\pi_{\star} \nu=\mu_{f}$, cette mesure 
est mélangeante. On travaillera dans l'espace $X:=\left\{\hat{x} \in \mathrm{O}: x_{n} \notin \mathcal{C}_{f}, \forall n \in \mathbb{Z}\right\}$ où $C_{f}$ désigne l'ensemble critique de $f$. Comme $\mu_{f}\left(\mathcal{C}_{f}\right)=0$, l'ensemble $X$ est de mesure pleine pour $\nu$. Pour tout $\hat{x} \in X$, on note $f_{\hat{x}}^{-n}$ la branche inverse de $f^{n}$ définie au voisinage de $x_{0}$ et envoyant $x_{0}$ sur $x_{-n}$. Rappelons qu'une fonction $\left.\left.\alpha: \mathrm{O} \rightarrow\right] 0,1\right]$ est dite $\epsilon$-lente si $\alpha \circ \tau \geq e^{-\epsilon} \alpha$.

On note $d(\cdot, \cdot)$ la distance induite sur $\mathbb{P}^{k}$ par la métrique de Fubini-Study, $B_{x}(r) \subset \mathbb{P}^{k}$ la boule centrée en $x$ et de rayon $r$ pour cette distance et $B(r)$ la boule $\{\|z\|<r\} \subset \mathbb{C}^{k}$ pour la distance hermitienne usuelle.

Le théorème 1.4 de [BDM] stipule que, pour $0<\epsilon \ll \chi_{1}$, il existe des fonctions $\epsilon$-lentes $r_{\epsilon}$, $\left.\left.t_{\epsilon}, 1 / \beta_{\epsilon}: X \rightarrow\right] 0,1\right]$, une constante $0<\alpha \leq 1$, des applications injectives $S_{\hat{x}}$ et des applications linéaires $R_{\widehat{x}}^{n}$ telles que le diagramme

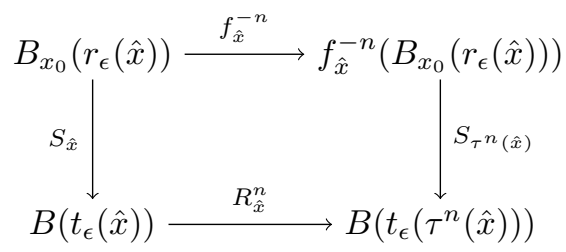

commute pour tout $n \in \mathbb{N}$ et tout $\hat{x} \in X$.

Soulignons que $S_{\hat{x}}\left(x_{0}\right)=0$ et que la linéarité des applications $R_{\hat{x}}^{n}$ découle de l'absence de résonnance sur les exposants de Lyapounov de $f$. En outre, les applications $S_{\hat{x}}$ et $R_{\hat{x}}^{n}$ satisfont les estimations suivantes

$$
\begin{gathered}
e^{-n \chi_{k}}\|z\| \leq\left\|R_{\hat{x}}^{n}(z)\right\| \leq e^{-n \chi_{1}}\|z\| \\
\alpha d(p, q) \leq\left\|S_{\tau^{n}(\hat{x})}(p)-S_{\tau^{n}(\hat{x})}(q)\right\| \leq \beta_{\epsilon}\left(\tau^{n}(\hat{x})\right) d(p, q) \\
e^{-n\left(\chi_{k}+\epsilon\right)} \frac{\alpha}{\beta_{\epsilon}(\hat{x})} d(p, q) \leq d\left(f_{\hat{x}}^{-n}(p), f_{\hat{x}}^{-n}(q)\right) \leq e^{-n \chi_{1}} \frac{\beta_{\epsilon}(\hat{x})}{\alpha} d(p, q)
\end{gathered}
$$

l'assertion (10) étant une conséquence directe de la commutativité du diagramme et des assertions (8) et (9).

Posons $\rho_{\epsilon}:=\alpha \frac{r_{\epsilon}}{\beta_{\epsilon}}$ (c'est une fonction $2 \epsilon$-lente telle que $0<\rho_{\epsilon} \leq r_{\epsilon}$ ) puis, pour tout $0<$ $t \leq 1$ définissons les ensembles $E_{\hat{x}}^{-n}(t) \subset \widetilde{E}_{\hat{x}}^{-n}(t)$ par $E_{\hat{x}}^{-n}(t):=f_{\hat{x}}^{-n}\left(B_{x_{0}}\left(t \rho_{\epsilon}(\hat{x})\right)\right)$ et $\widetilde{E}_{\hat{x}}^{-n}(t):=$ $f_{\hat{x}}^{-n}\left(B_{x_{0}}\left(\operatorname{tr}_{\epsilon}(\hat{x})\right)\right)$. L'objet du lemme suivant est de préciser la géométrie des ensembles $E_{\hat{x}}^{-n}(t)$.

Lemme 3.1. Pour tout $0<t \leq 1$ et toute paire de points $p, q$ de $E_{\hat{x}}^{-n}(t)$, il existe un chemin lisse joignant $p$ à $q$ dans $\widetilde{E}_{\hat{x}}^{-n}(t)$ et dont la longueur est majorée par $\frac{\beta_{\epsilon}(\hat{x})}{\alpha} e^{n \epsilon} d(p, q)$.

DÉmonstration: Si $p, q \in E_{\hat{x}}^{-n}(t)$ alors $f^{n}(p), f^{n}(q) \in B_{x_{0}}\left(t \rho_{\epsilon}(\hat{x})\right)$ et $R_{\hat{x}}^{n} \circ S_{\hat{x}}\left(f^{n}(p)\right)=S_{\tau^{n}(\hat{x})}(p)$, $R_{\hat{x}}^{n} \circ S_{\hat{x}}\left(f^{n}(q)\right)=S_{\tau^{n}(\hat{x})}(q)$. On voit ainsi, en utilisant (9), que

$$
S_{\tau^{n}(\hat{x})}(p), S_{\tau^{n}(\hat{x})}(q) \in R_{\hat{x}}^{n}\left[S_{\hat{x}}\left(B_{x_{0}}\left(t \rho_{\epsilon}(\hat{x})\right)\right)\right] \subset R_{\hat{x}}^{n}\left[B\left(t \alpha r_{\epsilon}(\hat{x})\right)\right] .
$$

Vérifions également que

$$
R_{\hat{x}}^{n}\left[B\left(t \alpha r_{\epsilon}(\hat{x})\right)\right] \subset S_{\tau^{n}(\hat{x})}\left[B_{x_{-n}}\left(\operatorname{tr}_{\epsilon}\left(\tau^{n}(\hat{x})\right)\right)\right] .
$$


Cela résulte des inclusions $R_{\hat{x}}^{n}\left[B\left(t \alpha r_{\epsilon}(\hat{x})\right)\right] \subset B\left(t \alpha r_{\epsilon}(\hat{x}) e^{-n \chi_{1}}\right) \subset B\left(t \alpha r_{\epsilon}(\hat{x}) e^{-n \epsilon}\right)$ et $B\left(t \alpha r_{\epsilon}(\hat{x}) e^{-n \epsilon}\right) \subset$ $B\left(\operatorname{tar}_{\epsilon}\left(\tau^{n}(\hat{x})\right)\right) \subset S_{\tau^{n}(\hat{x})}\left[B_{x_{-n}}\left(\operatorname{tr}_{\epsilon}\left(\tau^{n}(\hat{x})\right)\right)\right]$ qui se déduisent respectivement de (8) et (9).

On peut donc considérer l'image par $\left(S_{\tau^{n}(\hat{x})}\right)^{-1}$ du segment joignant $S_{\tau^{n}(\hat{x})}(p)$ à $S_{\tau^{n}(\hat{x})}(q)$ dans le convexe $R_{\hat{x}}^{n}\left[B\left(\operatorname{tar}_{\epsilon}(\hat{x})\right)\right]$. C'est un chemin joignant $p$ à $q$. En utilisant (9), on voit que sa longueur est majorée par $\frac{1}{\alpha} \beta_{\epsilon}\left(\tau^{n}(\hat{x})\right) d(p, q) \leq \frac{\beta_{\epsilon}(\hat{x})}{\alpha} e^{n \epsilon} d(p, q)$. Enfin, ce chemin est contenu dans $\widetilde{E}_{\hat{x}}^{-n}(t) \operatorname{car}\left(S_{\tau^{n}(\hat{x})}\right)^{-1}\left[R_{\hat{x}}^{n}\left[B\left(t \alpha r_{\epsilon}(\hat{x})\right)\right]\right] \subset\left(S_{\tau^{n}(\hat{x})}\right)^{-1} \circ R_{\hat{x}}^{n} \circ S_{\hat{x}}\left[B_{x_{0}}\left(\operatorname{tr}_{\epsilon}(\hat{x})\right)\right]=\widetilde{E}_{\hat{x}}^{-n}(t)$.

La Proposition suivante est obtenue en combinant les résultats décrits ci-dessus avec un argument classique dû à Briend-Duval.

Proposition 3.2. Soit $f \in \mathcal{H}_{d \geq 2}\left(\mathbb{P}^{k}\right)$. On suppose que les exposants de Lyapounov $\chi_{1} \leq \cdots \leq \chi_{k}$ de $f$ par rapport à sa mesure d'entropie maximale $\mu_{f}$ ne satisfont aucune relation de résonnance. Alors, pour tout ouvert $\Omega \subset \mathbb{P}^{k}$ tel que $\mu_{f}(\Omega)>0$ et tout $0<\epsilon \ll 1$, il existe des constantes $r_{0}, C, K>0$ (dépendant de $\epsilon$ ) ainsi qu'une boule $A \subset \Omega$ chargée par $\mu_{f}$ telles que, pour tout $n$ assez grand, $f^{n}$ admet $m \geq C d^{k n}$ branches inverses $g_{1}, \cdots, g_{m}$ définies sur des boules $B_{x_{0}^{j}}\left(K r_{0}\right)$ vérifiant $B_{x_{0}^{j}}\left(K r_{0}\right) \supset B_{x_{0}^{j}}\left(r_{0}\right) \supset A$ et

1) $g_{j}(A) \Subset A$,

2) $e^{-n\left(\chi_{k}+\epsilon\right)} \frac{1}{K} d(p, q) \leq d\left(g_{j}(p), g_{j}(q)\right) \leq e^{-n \chi_{1}} K d(p, q)$ sur $B_{x_{0}^{j}}\left(K r_{0}\right)$,

3) deux points quelconques $p, q$ de $g_{j}\left(B_{x_{0}^{j}}\left(r_{0}\right)\right)$ sont reliés par un chemin lisse dans $g_{j}\left(B_{x_{0}^{j}}\left(K r_{0}\right)\right)$ dont la longueur est majorée par $K e^{n \epsilon} d(p, q)$.

DÉmonstration: Rappelons que $\rho_{\epsilon}=\alpha \frac{r_{\epsilon}}{\beta_{\epsilon}}$ et définissons une partie $\widehat{H}$ de $X$ par $\widehat{H}:=\{\hat{x} \in X$ : $\left.r_{\epsilon}(\hat{x}) \geq K r_{0}, \frac{\alpha}{\beta_{\epsilon}(\hat{x})} \geq \frac{1}{K}\right\}$. Choisissons $K \gg 1$ puis $0<r_{0} \ll 1$ pour que $\nu(\widehat{H} \cap \widehat{\Omega})>0$.

Par un argument de recouvrement, on trouve une boule $A_{r}$ de rayon $r>0$ dans $\Omega$ et $0<\gamma \ll r$ tels que $r+\gamma<\frac{r_{0}}{2}$ et $\nu\left(\widehat{H} \cap \widehat{A}_{r+\gamma}\right)>0$. Observons que si $\hat{x} \in \widehat{H} \cap \widehat{A}_{r+\gamma}$ alors $A_{r+\gamma} \subset$ $B_{x_{0}}(2(r+\gamma)) \Subset B_{x_{0}}\left(r_{0}\right) \subset B_{x_{0}}\left(K r_{0}\right)$ et qu'alors $f_{\hat{x}}^{-n}$ est bien définie sur $A_{r+\gamma}$. On définit alors $C_{n}$ par $C_{n}:=\left\{\hat{x} \in \widehat{H} \cap \widehat{A}_{r+\gamma}: f_{\hat{x}}^{-n}\left(A_{r+\gamma}\right) \cap A_{r} \neq \emptyset\right\}$.

À chaque $\hat{x} \in C_{n}$ correspond une branche inverse $g:=f_{\hat{x}}^{-n}$ de $f^{n}$ définie sur une boule $B_{x_{0}}\left(K r_{0}\right) \supset$ $A_{r+\gamma}$. Il résulte du théorème 1.4 de $[\mathrm{BDM}$ (dont le contenu est rappelé ci-dessus) et de la définition de $\widehat{H}$ que $g$ satisfait les assertions (2) et (3). Plus précisément, l'assertion (2) est une reformulation de (10) tenant compte de $\frac{\alpha}{\beta_{\epsilon}(\hat{x})} \geq \frac{1}{K}$ et l'assertion (3) s'obtient en appliquant le Lemme 3.1 avec $t:=\frac{r_{0}}{\rho_{\epsilon}(\hat{x})}$. Il est utile d'observer que $t=\frac{r_{0}}{r_{\epsilon}(\hat{x})} \frac{r_{\epsilon}(\hat{x})}{\rho_{\epsilon}(\hat{x})} \leq \frac{1}{K} \frac{\beta_{\epsilon}(\hat{x})}{\alpha} \leq 1$ et que $\operatorname{tr}_{\epsilon}(\hat{x})=r_{0} \frac{r_{\epsilon}(\hat{x})}{\rho_{\epsilon}(\hat{x})}=$ $r_{0} \frac{\beta_{\epsilon}(\hat{x})}{\alpha} \leq K r_{0}$.

En particulier, puisque $g\left(A_{r+\gamma}\right) \cap A_{r} \neq \emptyset$ et diam $g\left(A_{r+\gamma}\right) \leq 2(r+\gamma) e^{-n \chi_{1}} K$, on voit que $g\left(A_{r+\gamma}\right) \Subset A_{r+\gamma}$ pourvu que $e^{n \chi_{1}}>2 \frac{r+\gamma}{\gamma} K$. On prendra donc $A:=A_{r+\gamma}$.

Il reste à établir l'assertion (1) et, pour cela, à minorer le nombre de branches inverses distinctes audessus de $A$ associées aux $\hat{x} \in C_{n}$. Notons $m$ ce nombre. Observons que deux éléments distincts de $C_{n}$ donnent lieu à des images $f_{\hat{x}}^{-n}(A)$ disjointes ou confondues. Nous allons combiner le caractère mélangeant de $\nu$ et le fait que le jacobien de $\mu$ est constant égal à $d^{k}$. La premiere propriété implique que

$$
\nu\left(\tau^{n}(\widehat{H} \cap \widehat{A}) \cap \widehat{A}_{r}\right) \rightarrow \nu(\widehat{H} \cap \widehat{A}) \cdot \nu\left(\widehat{A}_{r}\right)
$$


pour $n \rightarrow \infty$. En utilisant le fait que $f^{*} \mu=d^{k} \mu$ on a donc

$$
\begin{aligned}
m \mu(A) d^{-k n} & \geq \mu\left(\cup_{C_{n}} f_{\widehat{x}}^{-n}(A)\right) \\
& \geq \nu\left(\tau^{n}(\widehat{H} \cap \widehat{A}) \cap \widehat{A}_{r}\right) \geq \nu(\widehat{H} \cap \widehat{A}) \nu\left(\widehat{A}_{r}\right) / 2>0
\end{aligned}
$$

pour $n$ assez grand, ce qui donne l'estimation annoncée.

Considérons maintenant une famille holomorphe $F: D \times \mathbb{P}^{k} \rightarrow D \times \mathbb{P}^{k}$. Notons $f_{\lambda}:=F(\lambda, \cdot)$ l'endomorphisme de $\mathbb{P}^{k}$ correspondant au paramètre $\lambda$ et $\mu_{\lambda}$ sa mesure d'entropie maximale. Soit $\Omega \subset \mathbb{P}^{k}$ un ouvert chargé par $\mu_{0}$ et $g_{0,1}, \cdots, g_{0, m}$ les branches inverses de $f_{0}^{n}$ fournies par la Proposition 3.2. Nous montrerons que, quitte à diminuer légèrement $A$, les $g_{0, j}$ se prolongent en des biholomorphismes $G_{j}: D_{r(n)} \times A \rightarrow G_{j}\left(D_{r(n)} \times A\right)$ qui héritent des $g_{0, j}$ leurs propriétés contractantes. C'est ici que l'assertion (3) de la Proposition 3.2 et implicitement le théorème de linéarisation de $[\mathrm{BDM}]$ jouent un rôle crucial. Cela conduira à la proposition suivante.

Proposition 3.3. Soit $F: D \times \mathbb{P}^{k} \rightarrow D \times \mathbb{P}^{k}$ une famille holomorphe d'endomorphismes de degré $d \geq 2$. On suppose que les exposants de Lyapounov $\chi_{1} \leq \cdots \leq \chi_{k}$ de l'endomorphisme $f_{0}:=F(0, \cdot)$ par rapport à sa mesure d'entropie maximale $\mu_{0}$ ne satisfont aucune relation de résonnance. Alors, pour tout ouvert $\Omega \subset \mathbb{P}^{k}$ tel que $\mu_{0}(\Omega)>0$ et tout $0<\epsilon \ll 1$, il existe une constante $C>0$ (dépendant de $\epsilon$ ) ainsi qu'une boule $A \subset \Omega$ chargée par $\mu_{0}$ telles que, pour tout $n$ assez grand, $F^{n}$ admet $m \geq C d^{k n}$ branches inverses $G_{1}, \cdots, G_{m}$ définies au voisinage de $\overline{D_{r(n)}} \times \bar{A}$ et vérifiant

1) $G_{j}\left(D_{r(n)} \times A\right) \subset D_{r(n)} \times A, \forall 1 \leq j \leq m$,

2) $\operatorname{dist}\left(G_{j}\left(D_{r(n)} \times A\right), G_{k}\left(D_{r(n)} \times A\right)\right)>0, \forall 1 \leq j \neq k \leq m$,

3) $e^{-n\left(\chi_{k}+3 \epsilon\right)} d\left(z, z^{\prime}\right) \leq d\left(G_{j}(\lambda, z), G_{j}\left(\lambda, z^{\prime}\right)\right) \leq e^{-n\left(\chi_{1}-\epsilon\right)} d\left(z, z^{\prime}\right)$ sur $D_{r(n)} \times A$.

Démonstration: Rappelons que les branches $g_{0, j}$ données par la Proposition 3.2 sont toutes définies sur une même boule $\widetilde{A}$ (contenue dans $\Omega$ ) et qu'elles induisent des biholomorphismes $g_{0, j}: B_{x_{0}^{j}}\left(r_{0}\right) \rightarrow g_{0, j}\left(B_{x_{0}^{j}}\left(r_{0}\right)\right)$ où $\widetilde{A} \Subset B\left(x_{0}^{j}, r_{0}\right)$.

Soit $A$ une boule relativement compacte dans $\widetilde{A}$, obtenue en diminuant légèrement le rayon de $\widetilde{A}$ de façon à ce que $A$ reste chargée par $\mu_{0}$. Posons $A_{j}:=g_{0, j}(\widetilde{A})$, c'est un ouvert à bord régulier contenu dans $g_{0, j}\left(B_{x_{0}^{j}}\left(r_{0}\right)\right)$ et $f_{0}^{n}\left(b A_{j}\right)=b\left(f_{0}^{n}\left(A_{j}\right)\right)$. Les $f_{\lambda}^{n}$ étant continues et ouvertes, on a $b\left(f_{\lambda}^{n}\left(A_{j}\right)\right) \subset f_{\lambda}^{n}\left(b A_{j}\right)$ et l'on déduit alors de $A \Subset f_{0}^{n}\left(A_{j}\right)$ que $A \Subset f_{\lambda}^{n}\left(A_{j}\right)$ pour $\lambda \in D_{r(n)}$ pourvu que $r(n)$ soit assez petit. Cela se traduit par

$$
D_{r(n)} \times A \subset F^{n}\left(D_{r(n)} \times A_{j}\right) .
$$

Comme $f_{0}^{n}$ ne branche pas au voisinage de $\overline{A_{j}}$, on peut diminuer $r(n)$ de façon à ce que $F^{n}$ ne branche pas sur $D_{r(n)} \times A_{j}$. Soit $U_{j}$ une composante connexe de $\left(\left.F^{n}\right|_{D_{r(n)} \times A_{j}}\right)^{-1}\left(D_{r(n)} \times A\right)$, alors l'application $F^{n}: U_{j} \rightarrow D_{r(n)} \times A$ est holomorphe propre et ne branche pas. Puisque $D_{r(n)} \times A$ est simplement connexe c'est un biholomorphisme, on définit $G_{j}$ comme étant son inverse. Quitte à diminuer légèrement $r(n)$ et le rayon de $A$, on pourra supposer que $G_{j}$ est définie au voisinage de l'adhérence de $D_{r(n)} \times A$.

Voyons maintenant que les $G_{j}$ satisfont les estimations annoncées. Il faudra pour cela réduire à plusieurs reprises $r(n)$, ce que nous ferons sans le préciser. Les $G_{j}$ sont de la forme $G_{j}(\lambda, z)=$ $\left(\lambda, g_{\lambda, j}(z)\right)$ où $f_{\lambda}^{n} \circ g_{\lambda, j}=i d$. Puisque $g_{0, j}(A) \Subset A$ et $d\left(g_{0, j}(A), g_{0, k}(A)\right)>0$ si $j \neq k$ on a 
PERTURBATIONS D'EXEMPLES DE LATTÈS ET DIMENSION DE HAUSDORFF DU LIEU DE BIFURCATION9

$g_{\lambda, j}(A) \Subset A$ et $\operatorname{dist}\left(G_{j}\left(D_{r(n)} \times A\right), G_{k}\left(D_{r(n)} \times A\right)\right)>0$ si $j \neq k$.

Établissons l'estimation intermédiaire

$$
e^{-n\left(\chi_{k}+2 \epsilon\right)} \frac{1}{2 K^{2}} d\left(z, z^{\prime}\right) \leq d\left(G_{j}(\lambda, z), G_{j}\left(\lambda, z^{\prime}\right)\right) \leq e^{-n \chi_{1}} 2 K d\left(z, z^{\prime}\right) \text { sur } D_{r(n)} \times A .
$$

Par la Proposition [3.2, on a $d\left(g_{0, j}(z), g_{0, k}\left(z^{\prime}\right)\right) \leq K e^{-n \chi_{1}} d\left(z, z^{\prime}\right)$ pour $z, z^{\prime} \in B_{x_{0}^{j}}\left(r_{0}\right)$. Il s'ensuit que $\left\|d_{z} G_{j}(0, z)\right\| \leq e^{-n \chi_{1}} K$ sur $A$ puis que $\left\|d_{z} G_{j}(\lambda, z)\right\| \leq 2 e^{-n \chi_{1}} K$ sur $D_{r(n)} \times A$. Ceci entraîne la majoration dans (11).

Par la Proposition 3.2. on a $d\left(f_{0}^{n}(z), f_{0}^{n}\left(z^{\prime}\right)\right) \leq K e^{n\left(\chi_{k}+\epsilon\right)} d\left(z, z^{\prime}\right)$ pour tous $z, z^{\prime} \in g_{0, j}\left(B_{x_{0}^{j}}\left(r_{0}\right)\right)$. Il s'ensuit que $\left\|d_{z} f_{0}^{n}(z)\right\| \leq K e^{n\left(\chi_{k}+\epsilon\right)}$ sur $g_{0, j}\left(B_{x_{0}^{j}}\left(r_{0}\right)\right)$ et donc que $\left\|d_{z} F^{n}(\lambda, z)\right\| \leq 2 K e^{n\left(\chi_{k}+\epsilon\right)}$ sur $D_{r(n)} \times g_{0, j}\left(B_{x_{0}^{j}}\left(r_{0}\right)\right)$. Puisque $g_{0, j}(\widetilde{A}) \Subset g_{0, j}\left(B_{x_{0}^{j}}\left(r_{0}\right)\right)$ on a aussi $g_{\lambda, j}(A) \Subset g_{0, j}\left(B_{x_{0}^{j}}\left(r_{0}\right)\right)$ pour $\lambda \in D_{r(n)}$. Ainsi, compte tenu de la troisième assertion de la Proposition 3.2, si $(\lambda, z)$ et $\left(\lambda, z^{\prime}\right)$ sont dans $D_{r(n)} \times A$ alors les points $g_{\lambda, j}(z)$ et $g_{\lambda, j}\left(z^{\prime}\right)$ sont reliés par un chemin dont la longueur est majorée par $K e^{n \epsilon} d\left(g_{\lambda, j}(z), g_{\lambda, j}\left(z^{\prime}\right)\right)$ et sur lequel $\left\|d_{z} F^{n}(\lambda, z)\right\| \leq 2 K e^{n\left(\chi_{k}+\epsilon\right)}$. La minoration dans (11) s'ensuit car $d\left(z, z^{\prime}\right)=d\left(F^{n}\left(\lambda, g_{\lambda, j}(z)\right), F^{n}\left(\lambda, g_{\lambda, j}\left(z^{\prime}\right)\right)\right) \leq 2 K e^{n\left(\chi_{k}+\epsilon\right)} K e^{n \epsilon} d\left(g_{\lambda, j}(z), g_{\lambda, j}\left(z^{\prime}\right)\right)$. L'assertion (3) découle de (11) lorsque $n$ est assez grand.

\section{Dimension de Hausdorff du lieu de Bifurcation}

Considérons une famille holomorphe $F: M \times \mathbb{P}^{k} \rightarrow M \times \mathbb{P}^{k}$ d'endomorphismes de $\mathbb{P}^{k}$, notre objectif est d'obtenir des minorations locales de la dimension de Hausdorff de son lieu de bifurcation Bif. Nous allons pour cela utiliser les résultats des sections précédentes ainsi que la caractérisation suivante du lieu de bifurcation (voir le Théorème 1.6 de [BBD]).

Théorème 4.1. L'ensemble des paramètres du type Misiurewicz est une partie dense du lieu de bifurcation.

Définition 4.2. On dit que $\lambda_{0} \in D$ est un paramètre du type Misiurewicz si il existe une application holomorphe $\gamma$ définie sur un voisinage de $\lambda_{0}$ et à valeurs dans $\mathbb{P}^{k}$ telle que :

1) $\gamma\left(\lambda_{0}\right) \in J_{\lambda_{0}}$ et $\gamma(\lambda)$ est $p_{0}$-periodique répulsif pour $f_{\lambda}$ et un certain $p_{0} \geq 1$,

2) $\left(\lambda_{0}, \gamma\left(\lambda_{0}\right)\right) \in F^{n_{0}}\left(C_{F}\right)$ pour un certain $n_{0} \geq 1$,

3) le graphe $\Gamma_{\gamma}$ de $\gamma$ n'est pas contenu dans $F^{n_{0}}\left(C_{F}\right)$.

Rappelons que l'ensemble de Julia $J_{\lambda}$ de $f_{\lambda}:=F(\lambda, \cdot)$ est, par définition, le support de la mesure d'entropie maximale $\mu_{\lambda}$ de $f_{\lambda}$ et que $C_{F}$ désigne l'ensemble critique de l'application $F: M \times \mathbb{P}^{k} \rightarrow M \times \mathbb{P}^{k}$.

Le principal résultat de cette section est le suivant.

Proposition 4.3. Soit $F: D \times \mathbb{P}^{k} \rightarrow D \times \mathbb{P}^{k}$ une famille holomorphe d'endomorphismes de degré $d \geq 2$. On suppose que 0 est un paramètre Misiurewicz et que les exposants de Lyapounov $\chi_{1} \leq \cdots \leq \chi_{k}$ de $f_{0}:=F(0, \cdot)$ par rapport à sa mesure d'entropie maximale $\mu_{0}$ ne satisfont aucune relation de résonnance. On a alors l'estimation suivante : $\liminf _{r \rightarrow 0} \operatorname{dim}_{H}\left(D_{r} \cap \operatorname{Bif}\right) \geq$ $\frac{\chi_{1}}{\chi_{k}}\left(\frac{k \ln d}{\chi_{k}}\right)-(2 k-2)$.

Comme conséquence nous obtenons que la dimension de Hausdorff du lieu de bifurcation au voisinage d'un exemple de Lattès est maximale; c'est le théorème 1.3. 
DÉmonstration DU THÉORÈme 1.3: Notons $\chi_{1}(\lambda) \leq \cdots \leq \chi_{k}(\lambda)$ les exposants de Lyapounov de $f_{\lambda}$ et rappelons que $\chi_{1}(\lambda) \geq \frac{\ln d}{2}$. Que 0 soit dans le lieu de bifurcation résulte directement de la caractérisation des exemples de Lattès par la minimalité de leurs exposants et de celle de Bif comme étant le support de $d d^{c}\left(\chi_{1}(\lambda)+\cdots+\chi_{k}(\lambda)\right.$ ) (voir les Théorèmes 1.1 et 1.2), pour plus de détails nous renvoyons au Théorème 6.3 de [BBD]. En vertu du Théorème 4.1, on peut approcher 0 par des paramètres Misiurewicz $\lambda_{n}$. La somme des exposants de Lyapounov dépendant continûment du paramètre (voir [DS, Theorem 2.47] ) il vient $\lim _{n} \chi_{k}\left(\lambda_{n}\right)=\chi_{k}(0)=\frac{\ln d}{2}$ et $\lim _{n} \chi_{1}\left(\lambda_{n}\right)=\frac{\ln d}{2}$. En particulier les $\chi_{j}\left(\lambda_{n}\right)$ ne satisfont aucune relation de résonnance pour $n$ assez grand. La conclusion découle alors de la Proposition 4.3.

Remarque 4.4. En dimension $k=1$, l'exposant de Lyapounov $\chi(\lambda)$ est relié à la dimension de Hausdorff $\operatorname{dim}_{H} \mu_{\lambda}$ de la mesure $\mu_{\lambda}$ par la formule $\ln d=\chi(\lambda) \operatorname{dim}_{H} \mu_{\lambda}$. La Proposition 4.3 montre alors que pour toute famille holomorphe $F: D \times \mathbb{P}^{1} \rightarrow D \times \mathbb{P}^{1}$ de fractions rationnelles de degré $d \geq 2$ telle que 0 appartienne au lieu de bifurcation, on a $\liminf _{r \rightarrow 0} \operatorname{dim}_{H}\left(D_{r} \cap \operatorname{Bif}\right) \geq \operatorname{dim}_{H} \mu_{\lambda}$. Notons cependant que cette minoration est loin d'être optimale car, comme l'a montré M. Shishikura, la dimension de Hausdorff du bord de l'ensemble de Mandelbrot est égale à deux [Sh2].

DÉmonstration De la PROposition 4.3; Observons que l'on peut remplacer la famille $F$ par une itérée $F^{q}$ car le lieu de bifurcation ainsi que la quantité $\frac{\chi_{1}}{\chi_{k}}\left(\frac{k \ln d}{\chi_{k}}\right)$ apparaissant dans la minoration de $\operatorname{dim}_{H}\left(D_{r} \cap\right.$ Bif) restent alors inchangés. La démonstration reprend le principe de celle mise au point par le second auteur pour généraliser le Théorème 4.1 aux familles d'applications d'allure polynomiale [Bia]. Nous procéderons en cinq étapes.

Normalisations au voisinage d'un paramètre Misiurewicz. L'origine étant un para-mètre Misiurewicz, notons $\gamma$ la courbe holomorphe fournie par la Définition 4.2 On peut, quitte à renormaliser, supposer que $\gamma$ est définie sur $D$. En remplaçant $F$ par $F^{p_{0}}$, on peut aussi supposer que $p_{0}=1$.

En outre, une conjugaison par $(\lambda, z) \mapsto\left(\lambda, T_{\gamma(\lambda)}(z)\right)$ où $T_{\gamma(\lambda)}$ est une famille adéquate d'automorphismes de $\mathbb{P}^{k}$ permet de supposer que $\gamma$ est constant égal à $z_{1}:=\gamma(0)$. Désignons alors par $\Omega$ une boule centrée en $z_{1}$ et de rayon $r$. Si $\rho$ et $r$ sont pris assez petits, on a la situation suivante :

(i) $F$ est injective et uniformément expansive sur $D_{\rho} \times \Omega$ : il existe $K>1$ tel que

$$
\forall(\lambda, z) \in D_{\rho} \times \Omega, d\left(F(\lambda, z), F\left(\lambda, z_{1}\right)\right) \geq K d\left(z, z_{1}\right)
$$

(ii) $\left(\lambda, z_{1}\right) \in F^{n_{0}}\left(C_{F}\right) \Leftrightarrow \lambda=0$.

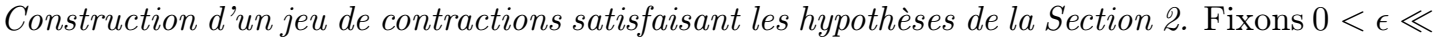
1 et appliquons la Proposition 3.3 à la famille $F$ et l'ouvert $\Omega$. (Rappelons que $\mu_{0}(\Omega)>0$ puisque $\left.z_{1} \in J_{0}\right)$. Quitte à renormaliser $D$, nous obtenons une boule $A$ contenue dans $\Omega$ et chargée par $\mu_{0}$ ainsi qu'une famille $G_{1}, \cdots, G_{m}$ de contractions holomorphes définies sur $\bar{D} \times \bar{A}$ et satisfaisant les estimations suivantes lorsque $n$ est assez grand :

1) $G_{j}(D \times A) \subset D \times A, \forall 1 \leq j \leq m$,

2) $\operatorname{dist}\left(G_{j}(D \times A), G_{k}(D \times A)\right)>0, \forall 1 \leq j \neq k \leq m$,

3) $e^{-n\left(\chi_{k}+3 \epsilon\right)} d\left(z, z^{\prime}\right) \leq d\left(G_{j}(\lambda, z), G_{j}\left(\lambda, z^{\prime}\right)\right) \leq e^{-n\left(\chi_{1}-\epsilon\right)} d\left(z, z^{\prime}\right)$ sur $D \times A$. 
Rappelons que $m$ et $n$ sont liés par l'inégalité $m \geq C d^{k n}$ et que l'on peut donc, quitte à choisir $n$ assez grand, supposer que

$$
\frac{\ln m}{n\left(\chi_{k}+3 \epsilon\right)} \geq \frac{k \ln d}{\chi_{k}+3 \epsilon}-\epsilon
$$

Dimininuer un peu $A$ et renormaliser $D$, permet de les remplacer par leurs adhérences dans les conditions 1),2),3) ci-dessus et d'assurer que les hypothèses de la Section 2 sont satisfaites.

Existence d'une courbe holomorphe de cycles $J$-répulsifs dans $D \times A$. Comme $\mu_{0}(A)>0$, il existe un point $z_{0} \in J_{0} \cap A$ qui est $p_{0}$-périodique et répulsif pour $f_{0}$ (d'après un théorème de BriendDuval $[\mathrm{BrDu}]$, ces points équidistribuent la mesure $\left.\mu_{0}\right)$. Quitte à renormaliser $D$, le théorème des fonctions implicites fournit une courbe holomorphe $\sigma: D \rightarrow A$ telle que $\sigma(0)=z_{0}$ et $\sigma(\lambda)$ est $p_{0}$-périodique et répulsif pour $f_{\lambda}$ pour tout $\lambda \in D$. Il reste à étudier l'appartenance de $\sigma(\lambda)$ à $J_{\lambda}$. Notons $B(\lambda)$ une boule centrée en $\sigma(\lambda)$ et de rayon $r>0$. On peut, quitte à renormaliser $D$ et diminuer $r$, supposer que $f_{\lambda}^{p_{0}}$ soit uniformément expansive sur $B(\lambda)$ pour tout $\lambda \in D$. Par ailleurs, $\sigma(0)$ appartenant à $J_{0}$ et $\lambda \mapsto J_{\lambda}$ étant s.c. $i$ pour la distance de Hausdorff, on peut renormaliser à nouveau $D$ de façon à ce que $B(\lambda) \cap J_{\lambda} \neq \emptyset$ pour tout $\lambda \in D$. Dans ces conditions, $\sigma(\lambda)$ est accumulé par des préimages par des itérées de $f_{\lambda}^{p_{0}}$ de points de $B(\lambda) \cap J_{\lambda}$ et donc, $J_{\lambda}$ étant fermé et totalement invariant, $\sigma(\lambda) \in J_{\lambda}$ pour tout $\lambda \in D$.

Mise en place des hypothèses de la Proposition 2.3. En utilisant la seconde étape et de la Section 2. on obtient une famille de graphes holomorphes $\mathcal{G}$ engendrée par la collection de contractions $G_{1}, \cdots, G_{m}$.

Le point $\left(0, z_{1}\right)$ étant dans $F^{n_{0}+n_{0}^{\prime}}\left(C_{F}\right)$ pour tout $n_{0}^{\prime} \in \mathbb{N}$, notons $Z_{0}$ la composante irreductible de $F^{n_{0}+n_{0}^{\prime}}\left(C_{F}\right) \cap(D \times \Omega)$ qui contient $\left(0, z_{1}\right)$. Il est clair que $Z_{0}$ n'est pas réduite à une fibre de $\pi_{D}$. En tenant compte des normalisations de la première étape, on voit que si $n_{0}^{\prime}$ est pris assez grand alors $\pi_{D}\left(Z_{0}\right) \Subset D$. Toute composante irreductible $Z$ de $Z_{0} \cap(D \times A)$ satisfait les hypothèses de la Proposition 2.3 et donne donc lieu à l'estimation

$$
\operatorname{dim}_{H} \pi_{D}(\mathcal{G} \cap Z) \geq \frac{\chi_{1}-\epsilon}{\chi_{k}+3 \epsilon}\left(\frac{\ln m}{n\left(\chi_{k}+3 \epsilon\right)}\right)-(2 k-2) .
$$

Avalanche de paramètres Misiurewicz et conclusion. Nous allons montrer que tous les éléments de $\pi_{D}(\mathcal{G} \cap Z)$ sont accumulés par des paramètres Misiurewicz et appartiennent donc, d'après le Théorèmes 4.1, au lieu de bifurcation. La conclusion résultera alors immédiatement des estimations (12) et (13) en faisant tendre $\epsilon$ vers 0 .

Nous utiliserons la courbe $\sigma$ exhibée à la troisième étape. Reprenons les notations de la Section 2 Par construction, tout $\Gamma_{\omega} \subset \mathcal{G}$ est limite, lorsque $p \rightarrow+\infty$, de la suite décroisantes $T_{\omega_{0} \cdots \omega_{p}}=G_{\omega_{0} \cdots \omega_{p}}(\bar{D} \times \bar{A})$. De plus cette convergence est uniforme (voir (1)). En particulier, la suite de graphes $G_{\omega_{0} \cdots \omega_{p}}\left(\Gamma_{\sigma}\right)$ converge vers $\Gamma_{\omega}$. Il s'ensuit que $G_{\omega_{0} \cdots \omega_{p}}\left(\Gamma_{\sigma}\right) \cap Z$ converge vers $\Gamma_{\omega} \cap Z$ et $\pi_{D}\left(G_{\omega_{0} \cdots \omega_{p}}\left(\Gamma_{\sigma}\right) \cap Z\right)$ vers $\pi_{D}\left(\Gamma_{\omega} \cap Z\right)$; d'après le Lemme 2.2 ces intersections sont non vides. Or les éléments de $\pi_{D}\left(G_{\omega_{0} \cdots \omega_{p}}\left(\Gamma_{\sigma}\right) \cap Z\right)$ sont des paramètres Misiurewicz car la non vacuité de $G_{\omega_{0} \cdots \omega_{p}}\left(\Gamma_{\sigma}\right) \cap Z$ signifie que $\Gamma_{\sigma}$ rencontre une composante de $F^{p+1+n_{0}+n_{0}^{\prime}}\left(C_{F}\right)$ sans y être contenu. 


\section{REFERENCES}

[BB] G. Bassanelli, F. Berteloot, Bifurcation currents in holomorphic dynamics on $\mathbb{C P}^{k}$, J. Reine Angew. Math., 608 (2007), 201-235.

[Ber] F. Berteloot, Bifurcation currents in holomorphic families of rational maps, Lecture Notes in Mathematics 2075 CIME Fundation subseries (2013) Springer Verlag, 1-93.

[BBD] F. Berteloot, F. Bianchi, C. Dupont, Dynamical stability and Lyapunov exponents for holomorphic endomorphisms of $C P(k)$, arXiv: 1403.7603 .

[BeDu] F. Berteloot, C. Dupont, Une caractérisation des exemples de Lattès par leur mesure de Green, Comment. Math. Helv., 80 (2005), no. 2, 433-454.

[BDM] F. Berteloot, C. Dupont, L. Molino, Poincaré-Dulac theorem for random families of contractions and applications to holomorphic dynamics, Ann. Inst. Fourier, 58 (2008), no. 6, 2137-2168.

[BL] F. Berteloot, J.-J. Loeb, Une caractérisation géométrique des exemples de Lattès de $\mathbb{C P}^{k}$, Bull. Soc. Math. Fr., 129 (2001), no. 2, 175-188.

[Bia] F. Bianchi, Motions of Julia sets and dynamical stability in several complex variables, PhD Thesis, Université Toulouse III Paul Sabatier and Università di Pisa, 2016.

[BiTa] F. Bianchi, J. Taflin, Bifurcations in the elementary Desboves family, Proc. of the AMS (2016), to appear.

[BrDu] J.-Y. Briend, J. Duval, Exposants de Liapounoff et distribution des points périodiques d'un endomorphisme de $\mathbb{P}^{k}$, Acta Math., 182 (1999), no. 2, 143-157.

[BuGa] X. Buff, T. Gauthier, Perturbation of flexible Lattès maps, Bull. Soc. Math. France, 141 (2013), no. 4, 603-614.

[deM] L. DeMarco, Dynamics of rational maps: Lyapunov exponents, bifurcations, and capacity, Math. Ann., 326 (2003), no. 1, 43-73.

[DS] T.-C. Dinh, N. Sibony, Dynamics in several complex variables: endomorphisms of projective spaces and polynomial-like mappings, Lecture Notes in Math. 1998 (2010).

[Duj] R. Dujardin, Non density of stability for holomorphic mappings on $P^{k}$ arXiv:1610.01785

[Dup] C. Dupont, Formule de Pesin et applications méromorphes, Bull. Braz. Math. Soc. (N.S.), 37 (2006), no. $3,393-418$

[Gau] T. Gauthier, Strong bifurcation loci of full Hausdorff dimension, Ann. Sci. Ecole Norm. Sup. $4^{\circ}$ série, 45 (2012), no. 6, 947-984.

[Lat] S. Lattès, Sur l'itération des substitutions rationelles et les fonctions de Poincaré 166, (1918), 26-28.

[Ly1] M. Lyubich, Some typical properties of the dynamics of rational mappings, Russian Math. Surveys, 38 (1983), no. 5, 154-155.

[Ly2] M. Lyubich, Investigation of the stability of the dynamics of rational functions, Teor. Funktsii Funktsional. Anal. i Prilozhen., 42 (1984), 72-91. Translated in Selecta Mathematica Sovietica, 9 (1990), no. 1, 69-90.

[MSS] R. Mañé, P. Sad, D. Sullivan, On the dynamics of rational maps, Ann. Sci. Ecole Norm. Sup. (4) 16 (1983), no. 2, 193-217.

[Mil] J. Milnor, On Lattès maps, Dynamics on the Riemann sphere, Eur. Math. Soc., Zürich (2006), 9-43.

[Pes] Y. Pesin, Dimension theory in dynamical systems, contemporary views and applications, Chicago Lectures in Mathematics (1997).

[PW] Y. Pesin, H. Weiss, On the dimension of deterministic and random Cantor-like sets, symbolic dynamics, and the Eckmann-Ruelle conjecture, Comm. Math. Phys. 182 (1996), no. 1, 105-153.

[Sh1] M. Shishikura, On the quasiconformal surgery of rational functions, Ann. Sci. Ecole Norm. Sup. (4) 20 (1987), no. 1, 1-29.

[Sh2] M. Shishikura, The Hausdorff dimension of the boundary of the Mandelbrot set and Julia sets, Ann. of Math. 147 (1998), no 2., 225-267.

[Zdu] A. Zdunik, Parabolic orbifolds and the dimension of the maximal measure for rational maps, Invent. Math., 99 (1990), no. 3, 627-649.

Université de Toulouse - IMT, UMR CNRS 5219, 31062 Toulouse Cedex, France

E-mail address: francois.berteloot@math.univ-toulouse.fr

Imperial College, South Kensington Campus, London SW7 2AZ, UK

E-mail address: f.bianchi@imperial.ac.uk 